\title{
Decision-Making Analysis for Hyperthermic Intraperitoneal Chemotherapy in Ovarian Cancer: A Survey by the Executive Committee of the Peritoneal Surface Oncology Group International (PSOGI)
}

\author{
Thomas Steffen $^{a} \quad$ Lukas Häller $^{\mathrm{a}} \quad$ Lana Bijelic $^{\mathrm{b}} \quad$ Markus Glatzer $^{\mathrm{c}}$ \\ Olivier Glehen $^{d}$ Diane Goérée ${ }^{\text {Ignace de Hingh }}{ }^{f}$ Yan $\mathrm{Li}^{\mathrm{g}}$ \\ Brendan J. Moran ${ }^{h}$ David L. Morris ${ }^{i}$ Pompiliu Piso ${ }^{j}$ Claudio A. Quadros ${ }^{k}$ \\ Beate Raul Paul Sugarbaker ${ }^{m}$ Yutaka Yonemuran Paul M. Putora ${ }^{\text {c, o }}$ \\ aDepartment of Surgery, Cantonal Hospital of St. Gallen, St. Gallen, Switzerland; beritoneal Surface Malignancies \\ Unit, Hospital Sant Joan Despi Moises Broggi, Barcelona, Spain; 'Department of Radiation Oncology, Cantonal \\ Hospital of St. Gallen, St. Gallen, Switzerland; 'Department of Digestive Surgery, Hospices Civils de Lyon, Lyon, \\ France; 'Department of Surgery, Gustave Roussy Institute, Villejuif, France; fDepartment of Surgery, Catharina \\ Hospital, Eindhoven, The Netherlands; ' Department of Peritoneal Cancer Surgery, Beijing Shijitan Hospital, \\ Beijing, PR China; hPeritoneal Malignancy Institute, Basingstoke and North Hampshire Hospital, Basingstoke, \\ UK; 'Department of Surgery, St. George Hospital and University of New South Wales, Sydney, NSW, Australia; \\ jDepartment of Surgical Oncology, Hospital Barmherzige Brüder Regensburg, Regensburg, Germany; ${ }^{\mathrm{k} S u r g i c a l}$ \\ Oncology Unit, São Rafael Hospital, Salvador, Brazil; 'Department of General Surgery, Charité - Universitätsmedizin \\ Berlin, Berlin, Germany; mPeritoneal Surface Oncology Unit, MedStar Washington Hospital Center, Washington,

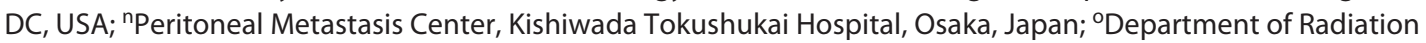 \\ Oncology, University of Bern, Bern, Switzerland
}

\section{Keywords}

Chemotherapy · Hyperthermic intraperitoneal chemotherapy · Metastasis · Ovarian cancer · Peritoneal carcinomatosis · Peritoneal malignancy

\section{Abstract \\ Objectives: To assess the individual treatment strategies among international experts in peritoneal carcinosis, specif- ically their decision-making in the process of patient selec- tion for hyperthermic intraperitoneal chemotherapy (HIPEC)}

in women suffering from ovarian cancer, to identify relevant decision-making criteria, and to quantify the level of consensus for or against HIPEC. Methods: The members of the executive committee of the Peritoneal Surface Oncology Group International (PSOGI) were asked to describe the clinical conditions under which they would recommend HIPEC in patients with ovarian cancer and to describe any disease or patient characteristics relevant to their decision. All answers were then merged and converted into decision trees. The decision trees were then analyzed by applying the objective consensus methodology. Results: Nine experts in karger@karger.com www.karger.com/ocl

(c) 2020 S. Karger AG, Basel

Karger ${ }^{\prime}=$
Thomas Steffen

Department of Surgery, Cantonal Hospital of St. Gallen Rorschacherstrasse 95

CH-9007 St. Gallen (Switzerland)

thomas.steffen@kssg.ch 
surgical oncology provided information on their multidisciplinary treatment strategy including HIPEC for patients with advanced ovarian cancer. Three of the total of 12 experts did not perform HIPEC. Five criteria relevant to the decision on whether HIPEC is performed were applied. In patients with resectable disease, a peritoneal cancer index $(\mathrm{PCI})<21$, and epithelial ovarian cancer without distant metastasis, consent was received by $75 \%$ to perform HIPEC for women suffering from recurrent disease. Furthermore, in the primary disease setting, consent was received by $67 \%$ to perform HIPEC according to the same criteria. Discussion and Conclusion: Among surgical oncology experts in peritoneal surface malignancy and HIPEC, HIPEC plays an important role in primary and recurrent ovarian cancer, and the $\mathrm{PCl}$ is the most important criterion in this decision.

(c) 2020 S. Karger AG, Basel

\section{Introduction}

Ovarian cancer is the fifth leading cause of death from cancer among American women and the second most common gynecologic malignancy in developed countries, with an estimated age-standardized incidence of 9.1 per 100,000 [1]. Ovarian cancer is associated with the highest mortality of all gynecologic cancers (5.0 per 100,000) [2]. Most women suffering from ovarian cancer are diagnosed with advanced stage IIIC or IV disease according to the International Federation of Gynecology and Obstetrics (FIGO) classification [3]. In FIGO stage IIIC, the tumor has spread beyond the pelvis with peritoneal metastases of $>2 \mathrm{~cm}$ in dimension [3]. The standard treatment for primary disease is cytoreductive surgery (CRS) followed by systemic platinum-based chemotherapy [4]. Neoadjuvant chemotherapy has also been established and is found in clinical practice guidelines [4-6]. The ultimate goal of the surgical procedure is to achieve a so-called complete cytoreduction, which is of utmost relevance concerning prognosis [7]. The completeness of cytoreduction was defined according to the completeness of cytoreduction score (CC score) [8]. A combined exploratory analysis of 3 prospectively randomized phase III multicenter trials with 3,126 patients showed improved median recurrence-free and overall survival rates if a complete cytoreduction was achieved compared to patients with residual macroscopic tumor by 5.4 and 62.9 months, respectively [9].

With standard treatment, most patients experience a response to initial treatment. However, the long-term cure rates between 20 and $25 \%$ are daunting, and $80 \%$ of the patients recur $[10,11]$. The majority of recurrences are intraperitoneal; thus, locoregional therapy is a preferred and logical therapeutic concept [12].

The combination of intraperitoneal and systemic chemotherapy showed prolonged overall survival but has not been widely adopted as a standard of care because of concerns about treatment-related toxicities and other reasons, such as catheter-related complications [13].

Despite numerous published reviews and cohort studies on CRS and hyperthermic intraperitoneal chemotherapy (HIPEC), there has been limited evidence from phase III trials to definitively determine any survival benefit associated with CRS and HIPEC in patients with advanced ovarian cancer [14]. A recent multicenter, open-label, phase III trial with 245 patients showed that the addition of HIPEC following complete CRS is associated with prolonged recurrence-free and overall survival compared to surgery alone, without higher rates of side effects. The median recurrence-free survival and median overall survival in this study were prolonged by 3.5 and 11.8 months, respectively [15]. The results of this study are highly debated, and HIPEC following complete CRS is controversial. On the contrary, some professional societies explicitly do not recommend this treatment strategy.

When evidence does not provide clear guidance, clinical practice can vary substantially $[16,17]$. The consequence is that for the same disease entity, confirmed experts in the field may consider varying decision criteria and offer different treatment strategies to patients. Decision tree-based analyses have been applied in various cancer settings, including HIPEC for colorectal cancer [18-21]. In such situations, learning what is being performed in the community may provide valuable insight [22]. Expressing the clinical decision criteria of confirmed experts in a certain field in structured decision trees can serve as a basis to find and quantify discrepancies and consensus $[23,24]$.

There is often a discrepancy between guideline recommendations and their practical clinical implementation. The aim of this study was to assess the individual treatment strategies among international experts in peritoneal carcinosis, specifically their decision-making in the process of patient selection for HIPEC in women suffering from ovarian cancer. Our aim was to identify relevant decision criteria, to understand their interaction, and to quantify the level of consensus for or against HIPEC among experts.
Steffen et al. 
Fig. 1. Sample decision tree illustrating the answers from one expert for further analyses. In this tree, only 2 criteria were applied by the expert. OC, ovarian cancer; $\mathrm{M}$, distant metastases; PCI, peritoneal cancer index; CRS, cytoreductive surgery; HIPEC, hyperthermic intraperitoneal chemotherapy.

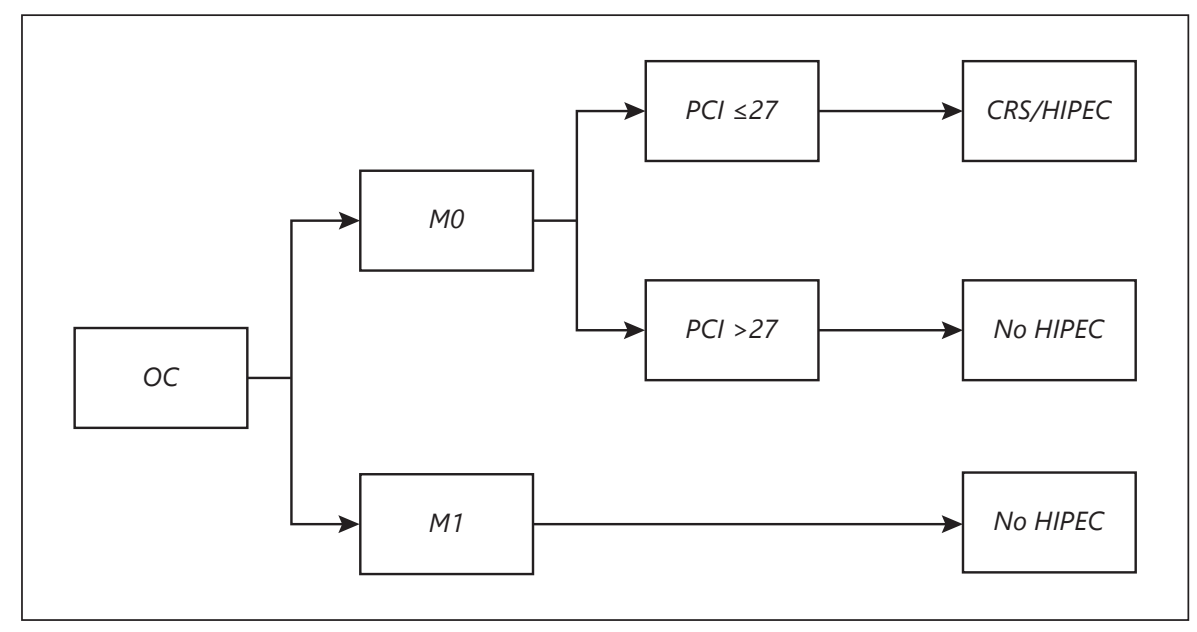

\section{Materials and Methods}

All members of the executive committee of the Peritoneal Surface Oncology Group International (PSOGI) were considered confirmed experts in the field and asked to participate in this analysis (www.psogi.com). All experts are specially trained and experienced in the field of CRS and HIPEC and perform up to several hundred HIPEC procedures per year. The experts were asked to describe the clinical conditions under which they would recommend HIPEC for patients with ovarian cancer. In addition, the participants were asked to describe any disease or patient characteristics relevant to their decision. To avoid diminishing the wide spectrum of opinions, answers were accepted in any form (e.g., free text, diagrams, decision trees, and tables). To avoid influencing the responses, no specific clinical scenarios were predefined, and the initial questions asked were open: "Please describe the circumstances under which you would recommend HIPEC in patients with ovarian cancer? Please describe any disease or patient characteristics relevant to your decision." Follow-up questions were asked to clarify the given answers and to avoid misunderstandings. All answers were then merged and converted into decision trees as described elsewhere [24].

To achieve consent and simplify the decision trees, criteria that were mentioned only once were excluded from the decision trees. Confirmed chemosensitivity was considered a generally accepted precondition to perform HIPEC by all experts and was therefore not included in the analysis.

The term "resectable" was defined as a completeness of cytoreduction score of CC-0 or CC-1 [8]. "Not resectable" was defined as more than CC-1 or in cases of tumor progression under neoadjuvant chemotherapy.

Universally accepted prerequisites for performing HIPEC, such as the general operability of the patient based upon performance status, comorbidities or other contraindications to surgery, extensive bowel disease, and infiltration of the root of the mesentery, were not represented in the analysis, otherwise the decision trees would have grown in complexity, making meaningful results almost impossible. The resulting decision trees were graphically finalized and reconfirmed by each expert by October 2019. The decision trees were analyzed by applying the objective consensus methodology [23].

Patient Selection for HIPEC in Ovarian Cancer Patients
Table 1. Relevant decision criteria per expert (A-I)

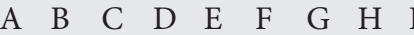

Primary/recurrent disease

CC-score

Distant metastases

PCI

Histology

Shaded areas indicate an accepted decision criterion. CC-score, completeness of cytoreduction score; PCI, peritoneal cancer index,

\section{Results}

Twelve out of 15 experts agreed to participate and were provided with written information on the interdisciplinary treatment strategy for patients with ovarian cancer and peritoneal carcinomatosis. Three of the 12 experts did not perform HIPEC in this setting, because this procedure was not approved or adequately reimbursed in the respective countries. Therefore, a total of 9 answers were available for further analysis.

For further analysis, 5 decision criteria relevant to the decision as to whether HIPEC is performed were applied (Table 1): (1) primary or recurrent disease, (2) CC-score, (3) presence of distant metastases, (4) peritoneal cancer index (PCI), and (5) histology. Per participating expert, up to all of these 5 criteria were considered relevant to the decision. As an exception, 1 expert accepted up to 3 resectable liver metastases to perform HIPEC. All other experts considered distant metastases a contraindication to performing HIPEC (Fig. 1). The presence of ascites, FIGO 


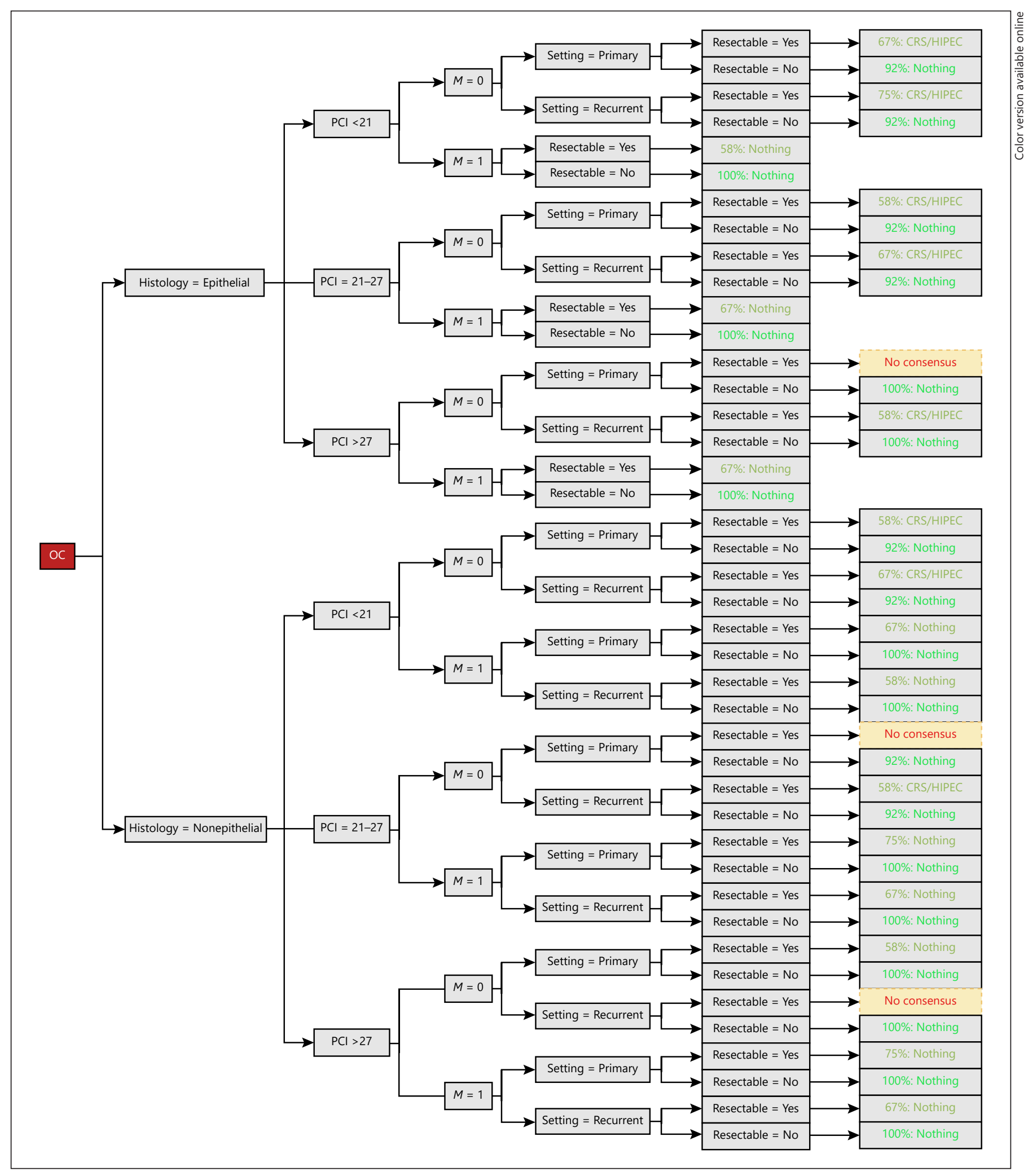

Fig. 2. Final decision tree showing the level of consensus for or against HIPEC after complete cytoreduction. Consensus was defined as a level of $50 \%$ or higher. OC, ovarian cancer; M, distant metastases; PCI, peritoneal cancer index; CRS, cytoreductive surgery; HIPEC, hyperthermic intraperitoneal chemotherapy. 


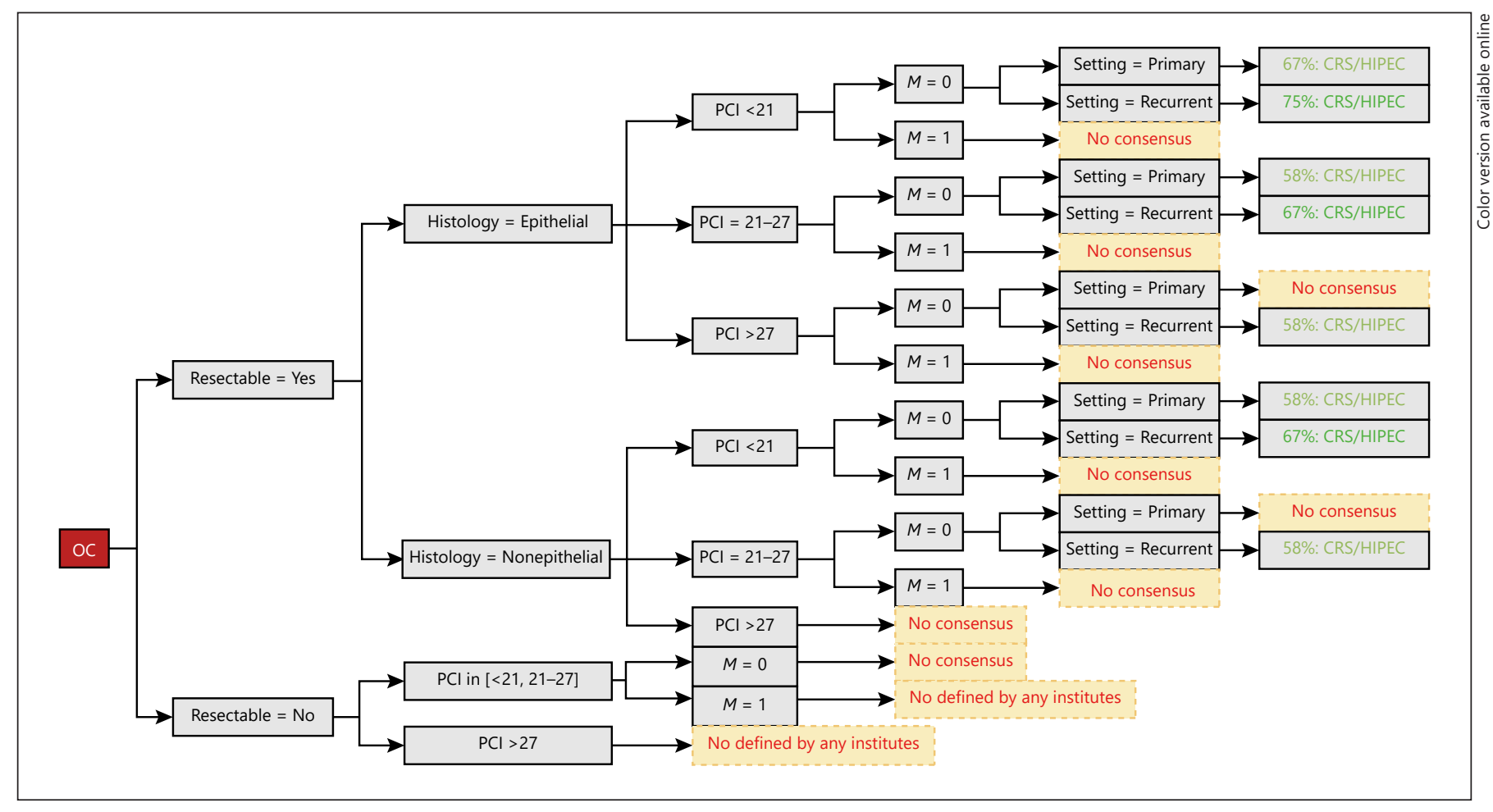

Fig. 3. Decision tree showing the levels of consent in favor of performing HIPEC. OC, ovarian cancer; M, distant metastases; PCI, peritoneal cancer index; CRS, cytoreductive surgery; HIPEC, hyperthermic intraperitoneal chemotherapy.

staging, age younger than 75 years, and multidisciplinary team recommendation were mentioned only once and are not represented in the decision tree analysis.

In patients with resectable disease, a $\mathrm{PCI}<21$, and epithelial ovarian cancer without distant metastasis, consent was received by $75 \%$ in favor of performing HIPEC on women suffering from recurrent disease. Furthermore, in primary disease, consent was received by $67 \%$ in favor of performing HIPEC with the same criteria. If the histology was nonepithelial, then $67 \%$ recommended HIPEC in recurrent disease and 58\% recommended HIPEC in primary disease, again applying the same criteria.

There was wide consent (100\%) to not performing HIPEC on patients with nonresectable disease, a PCI $>27$, and distant metastases. However, 1 expert (8\%) recommended HIPEC in this situation if the PCI is $<21$.

There was broad consensus on not performing HIPEC on patients with nonresectable peritoneal carcinomatosis in the presence of distant metastasis, independent of histology, PCI, and primary or recurrent disease. Only 1 expert would make an exception for up to 3 resectable liver metastases (Fig. 2).

Patient Selection for HIPEC in Ovarian Cancer Patients
Figure 3 depicts the aggregated levels of consent in favor of performing HIPEC.

\section{Discussion and Conclusion}

This is a decision-making analysis among surgical oncologists highly experienced in the field of CRS in patients with peritoneal malignancies. Our findings suggest that most of the experts recommend performing HIPEC on women suffering from recurrent disease if the tumor is considered resectable and the PCI is $<21$. Furthermore, two-thirds of the experts recommend performing HIPEC on women with primary ovarian cancer by applying the same criteria.

Thus, HIPEC may play an important role in the treatment of both primary and recurrent epithelial ovarian cancer. Similar recommendations have been made based on the most recent randomized controlled study by van Driel et al. [15], and multiple cohort studies have been published showing favorable survival data leading to recommendations that HIPEC be performed in ovarian cancer [25]. However, HIPEC for the treatment of ovarian 
cancer is very controversial in the field of gynecologic oncology. The above-mentioned study had been questioned and criticized in the same journal [26]. In relation to the findings of previous studies, our findings showed one clear PCI threshold of a maximum of 20 for the decision as to whether to perform HIPEC. The PCI as a criterion for this treatment has been under discussion for years, and the recommended thresholds have gradually moved to lower PCI values. One actual PCI threshold that has been advocated is a maximum of 18 points, and - not surprisingly - the lower the threshold was, the more favorable the oncologic outcome. A recent study found that the addition of HIPEC to CRS does not negatively impact the health-related quality of life [27], and two recent studies showed CRS and HIPEC to be cost-effective in two different countries [28, 29].

In total, 5 criteria relevant to the indication to perform HIPEC were found: (1) whether it is a primary tumor or recurrent disease; (2) whether the tumor is resectable, achieving a CC-score of $0 / 1$ (no or residual tumor masses of $\leq 2.5 \mathrm{~mm}$ ); (3) absence of distant metastases; (4) the PCI; and (5) the type of histology. Three of the 12 experts did not perform HIPEC on ovarian cancer patients, because this technique is not adequately reimbursed or approved in the respective countries, which obviously can be a criterion for not performing a treatment.

As an explanation for our results, we consider the clinical expertise involved here to be very high. The present study is not a patterns-of-care survey but a decision-making analysis. While some experts set their individual PCI threshold even higher, a relatively high maximal PCI threshold of 20 is still accompanied by a relevant amount of disease that needs special surgical experience for CRS to be successful. This surgical expertise can be expected within this group of specialized surgical oncologists. Furthermore, we were able to confirm by consent that the tumor has to be resectable by CRS down to residual tumor masses of $2.5 \mathrm{~mm}$ or smaller, and that the absence of distant metastases is a precondition for the performance of HIPEC on these women.

We consider our results to be clinically relevant, since HIPEC in ovarian cancer has been highly debated for years. In particular, the community of surgical gynecologists is not in agreement with this treatment option, and great reservations have been expressed, leading to several of them offering clear advice against HIPEC [30]. This discrepancy is comprehensibly due to a lack of high-quality studies, and, therefore, data clearly favoring HIPEC for these patients are lacking. However, the above-mentioned randomized controlled trial has been published, and we cannot disregard the results. Clearly, the results of that study also have limitations, as published elsewhere $[26,31]$. A recent Korean trial found that HIPEC had no positive influence on the oncologic outcome in patients with primary disease [32]. It remains challenging to interpret these results and to determine what they mean for clinical routine. We think that our findings can contribute to the acceptance of therapeutic concepts. For now, HIPEC in ovarian cancer patients is recommended within clinical trials only.

This study has some limitations. First, the experts involved are specialized in the field of surgery for peritoneal malignancies and are highly experienced and trained in CRS and HIPEC. This level of expertise may not represent the surgical or gynecologic expertise in general health care. Furthermore, since explicit experts in the field were asked, the consensual thresholds found may be higher than those in other samples. The number of experts involved in this study lowers the representability of the results; however, as a large heterogeneity was identified among this group, we would not expect significantly different results with a different subset of PSOGI board members. However, to provide broader support for the conclusions and pieces of advice derived from the present consensus findings and their acceptance in a broader community, the results could be evaluated in a future classic patterns-of-care survey. All variations in multimodal therapeutic concepts - for example, regimens of systemic chemotherapy - were omitted in this study; however, these concepts were also not mentioned as criteria for or against HIPEC. While the participating experts advocated HIPEC as a treatment concept, the results of this analysis may not be applicable to each individual patient. The selection of HIPEC experts poses the risk of a specialty bias toward HIPEC. Further research should include randomized controlled trials to define clear PCI thresholds and medication regimens for HIPEC concerning the whole multimodal treatment strategy.

We conclude that HIPEC in primary and recurrent ovarian cancer plays an important role among surgical oncologists specialized in peritoneal surface malignancy and HIPEC, and the PCI is the most important criterion in this decision.

\section{Statement of Ethics}

Ethical approval was not required, since no subjects were involved.
Steffen et al. 


\section{Conflict of Interest Statement}

The authors have no conflicts of interest do declare.

\section{Funding Sources}

There are no funding sources to declare.

\section{Author Contributions}

T.S.: study design, data acquisition, and writing of the manuscript; L.H.: data collection, data analyses, and writing of the manuscript; L.B.: medical expertise, critical revision of the manuscript, and final approval; M.G.: data analyses; O.G.: medical expertise, critical revision of the manuscript, and final approval; I.d.H.: medical expertise; critical revision of the manuscript, and final approval; Y.L.: medical expertise, critical revision of the manuscript, and final approval; B.J.M.: medical expertise, critical revision of the manuscript, and final approval; D.L.M.: medical expertise, critical revision of the manuscript, and final approval; P.P.: medical expertise, critical revision of the manuscript, and final approval; C.A.Q.: medical expertise, critical revision of the manuscript, and final approval; B.R.: medical expertise, critical revision of the manuscript, and final approval; P.S.: medical expertise, critical revision of the manuscript, final approval, and support of the publication process; Y.Y.: medical expertise, critical revision of the manuscript, and final approval; P.M.P.: study design, methodological expertise, critical revision of the manuscript, final approval, and support of the publication process.

\section{References}

1 Siegel RL, Fedewa SA, Miller KD, GodingSauer A, Pinheiro PS, Martinez-Tyson D, et al. Cancer statistics for Hispanics/Latinos, 2015. CA Cancer J Clin. 2015;65(6):457-80.

2 Torre LA, Bray F, Siegel RL, Ferlay J, LortetTieulent J, Jemal A. Global cancer statistics, 2012. CA Cancer J Clin. 2015;65(2):87-108.

3 Prat J. Staging classification for cancer of the ovary, fallopian tube, and peritoneum: abridged republication of guidelines from the International Federation of Gynecology and Obstetrics (FIGO). Obstet Gynecol. 2015; 126(1):171-4.

4 Cortez AJ, Tudrej P, Kujawa KA, Lisowska KM. Advances in ovarian cancer therapy. Cancer Chemother Pharmacol. 2018;81(1) $17-38$.

5 Vergote I, Tropé CG, Amant F, Kristensen GB, Ehlen T, Johnson N, et al. Neoadjuvant chemotherapy or primary surgery in stage IIIC or IV ovarian cancer. N Engl J Med. 2010; 363(10):943-53.

6 Wright AA, Bohlke K, Armstrong DK, Bookman MA, Cliby WA, Coleman RL, et al. Neoadjuvant chemotherapy for newly diagnosed, advanced ovarian cancer: Society of Gynecologic Oncology and American Society of Clinical Oncology Clinical Practice Guideline. Gynecol Oncol. 2016;143(1):3-15.

7 Sugarbaker PH. Peritonectomy procedures. Ann Surg. 1995;221(1):29-42.

8 Sugarbaker PH. Intraperitoneal chemotherapy and cytoreductive surgery for the prevention and treatment of peritoneal carcinomatosis and sarcomatosis. Semin Surg Oncol. 1998;14(3):254-61.

9 du Bois A, Reuss A, Pujade-Lauraine E, Harter P, Ray-Coquard I, Pfisterer J. Role of surgical outcome as prognostic factor in advanced epithelial ovarian cancer: a combined exploratory analysis of 3 prospectively randomized phase 3 multicenter trials: by the Arbeitsgemeinschaft Gynaekologische Onkologie Stu- diengruppe Ovarialkarzinom (AGO-OVAR) and the Groupe d'Investigateurs Nationaux Pour les Etudes des Cancers de l'Ovaire (GINECO). Cancer. 2009;115(6):1234-44.

10 Morgan RJ Jr., Alvarez RD, Armstrong DK, Burger RA, Chen LM, Copeland L, et al. Ovarian cancer, version 2.2013. J Natl Compr Canc Netw. 2013;11(10):1199-209.

11 Foley OW, Rauh-Hain JA, del Carmen MG. Recurrent epithelial ovarian cancer: an update on treatment. Oncology (Williston Park). 2013;27(4):288-94, 298.

12 Bakrin N, Classe JM, Pomel C, Gouy S, Chene G, Glehen O. Hyperthermic intraperitoneal chemotherapy (HIPEC) in ovarian cancer. J Visc Surg. 2014;151(5):347-53.

13 Wright AA, Cronin A, Milne DE, Bookman MA, Burger RA, Cohn DE, et al. Use and effectiveness of intraperitoneal chemotherapy for treatment of ovarian cancer. J Clin Oncol. 2015;33(26):2841-7.

14 Hotouras A, Desai D, Bhan C, Murphy J, Lampe B, Sugarbaker PH. Heated IntraPEritoneal Chemotherapy (HIPEC) for patients with recurrent ovarian cancer: a systematic literature review. Int J Gynecol Cancer. 2016; 26(4):661-70.

15 Driel WJ, Koole SN, Sikorska K, Schagen van Leeuwen JH, Schreuder HWR, Hermans RHM, et al. Hyperthermic intraperitoneal chemotherapy in ovarian cancer. $\mathrm{N}$ Engl J Med. 2018;378(3):230-40.

16 Glatzer M, Panje CM, Sirén C, Cihoric N, Putora PM. Decision making criteria in oncology. Oncology. 2020;98(6):370-8.

17 Panje CM, Glatzer M, Sirén C, Plasswilm L, Putora PM. Treatment options in oncology. JCO Clin Cancer Inform. 2018;2:1-10.

18 Rothermundt C, Bailey A, Cerbone L, Eisen T, Escudier B, Gillessen S, et al. Algorithms in the first-line treatment of metastatic clear cell renal cell carcinoma - analysis using diagnostic nodes. Oncologist. 2015;20(9):1028-35.
19 Hundsberger T, Hottinger AF, Roelcke U, Roth P, Migliorini D, Dietrich PY, et al. Patterns of care in recurrent glioblastoma in Switzerland: a multicentre national approach based on diagnostic nodes. J Neurooncol. 2016;126(1):175-83.

20 Rothermundt C, Fischer GF, Bauer S, Blay JY, Grünwald V, Italiano A, et al. Pre- and postoperative chemotherapy in localized extremity soft tissue sarcoma: a European Organization for Research and Treatment of Cancer expert survey. Oncologist. 2018; 23(4):461-7.

21 Steffen T, Putora PM, Hübner M, Gloor B, Lehmann K, Kettelhack C, et al. Diagnostic nodes of patient selection for cytoreductive surgery and hyperthermic intraperitoneal chemotherapy among colorectal cancer patients: a Swiss national multicenter survey. Clin Colorectal Cancer. 2019;18(4): e33542.

22 Putora PM, Oldenburg J. Swarm-based medicine. J Med Internet Res. 2013;15(9): e207.

23 Putora PM, Panje CM, Papachristofilou A, Dal Pra A, Hundsberger T, Plasswilm L. Objective consensus from decision trees. Radiat Oncol. 2014;9:270.

24 Panje CM, Glatzer M, von Rappard J, Rothermundt C, Hundsberger T, Zumstein V, et al. Applied swarm-based medicine: collecting decision trees for patterns of algorithms analysis. BMC Med Res Methodol. 2017;17(1): 123.

25 Huo YR, Richards A, Liauw W, Morris DL. Hyperthermic intraperitoneal chemotherapy (HIPEC) and cytoreductive surgery (CRS) in ovarian cancer: a systematic review and metaanalysis. Eur J Surg Oncol. 2015;41(12):157889.

26 Vergote I, Chiva L, du Bois A. Hyperthermic intraperitoneal chemotherapy in ovarian cancer. N Engl J Med. 2018;378(14):1362-3. 
27 Koole SN, Kieffer JM, Sikorska K, Schagen van Leeuwen JH, Schreuder HWR, Hermans $\mathrm{RH}$, et al. Health-related quality of life after interval cytoreductive surgery with or without hyperthermic intraperitoneal chemotherapy (HIPEC) in patients with stage III ovarian cancer. Eur J Surg Oncol. 2019 [Online ahead of print].

28 Lim SL, Havrilesky LJ, Habib AS, Secord AA. Cost-effectiveness of hyperthermic intraperitoneal chemotherapy (HIPEC) at interval debulking of epithelial ovarian cancer following neoadjuvant chemotherapy. Gynecol Oncol. 2019;153(2):376-80.
29 Koole SN, van Lieshout C, van Driel WJ, van Schagen E, Sikorska K, Kieffer JM, et al. Cost effectiveness of interval cytoreductive surgery with hyperthermic intraperitoneal chemotherapy in stage III ovarian cancer on the basis of a randomized phase III trial. J Clin Oncol. 2019;37(23):2041-50.

30 German Guideline Program in Oncology (GGPO). S3-Leitlinie Diagnostik, Therapie und Nachsorge maligner Ovarialtumoren. 2019 ;
31 Fotopoulou C, Sehouli J, Mahner S, Harter P, Van Nieuwenhuysen E, Gonzalez-Martin A, et al. HIPEC: HOPE or HYPE in the fight against advanced ovarian cancer? Ann Oncol. 2018;29(8):1610-3.

32 Lim MC, Chang SJ, Yoo HJ, Nam BH, Bristow $\mathrm{R}$, Park SY. Randomized trial of hyperthermic intraperitoneal chemotherapy (HIPEC) in women with primary advanced peritoneal, ovarian, and tubal cancer. J Clin Oncol. 2017; 35(15_Suppl):5520. 